\title{
Minnesota Policy on Pharmacist-Prescribed Contraceptives: Benefits, Limitations, and Opportunities for Improvement
}

\author{
Federico Facciolo, MS, PhD Student; Joel Farley, PhD, Professor
}

College of Pharmacy, University of Minnesota

\begin{abstract}
In August 2020, pharmacists were authorized to prescribe contraceptives in Minnesota outside of a collaborative practice agreement. To practice under this new authorization, pharmacists must complete formal contraceptive prescribing training and follow guidelines which include restrictions on patient's age and appropriate screening and assessment requirements. Allowing pharmacists to prescribe contraceptives has the potential to extend contraceptive access, decrease overall health care costs, and improve outcomes. However, barriers to the expansion of contraceptive prescribing by pharmacists may prevent widespread adoption of this practice in Minnesota. A key concern among pharmacists is a lack of reimbursement for providing this service. Other states adopting contraceptive prescribing by pharmacists which have not paid for this service have reported limited implementation and discontinuation of this service. To improve contraceptive access to Minnesotans, policymakers should consider expanding provider status to pharmacists and providing reimbursement to pharmacists for contraceptive services.
\end{abstract}

Keywords: Pharmacist Prescribing; Contraception; Community Pharmacy; Pharmacy Services; Pharmacy Policy; Access to Care; Birth Control; Public Health Services; Pharmacy Legislation

\section{Description of the policy}

In 2020, pharmacists were granted the authority to independently prescribe contraceptives in Minnesota outside of a collaborative practice agreement. ${ }^{1}$ The legislation went into effect on August 1st, 2020 and expanded prescriptive authority for contraceptives to pharmacists completing formal training on contraceptive prescribing through a college of pharmacy or another program approved by the Accreditation Council for Pharmacy Education or Minnesota Board of Pharmacy. ${ }^{2}$ Within the training prerequisites, pharmacists can prescribe contraceptives only for the intended use of contraception. In addition to defining requirements for pharmacist training to provide contraceptive services, this legislation also called on the Minnesota Board of Pharmacy to develop a protocol guiding contraceptive pharmacistprescribing practices. The protocol was created by the board in conjunction with agencies and professional associations, including the Minnesota section of the American Congress of Obstetricians and Gynecologists. ${ }^{3}$

The protocol developed by the Board of Pharmacy includes restrictions on the age of patients who can receive this service, limits on refills, patient screening and assessment requirements, prescribing algorithms, and requirements for patient counseling, referrals, delegation prohibitions, and prescription records. ${ }^{3}$ First, pharmacists are restricted to prescribing new contraceptives to patients aged 18 and older.

Corresponding author: Federico Facciolo, MS, PhD Student

College of Pharmacy

University of Minnesota

Minneapolis, MN

Email: facci010@umn.edu
Patients under the age of 18 may only utilize a pharmacist to prescribe contraceptives if there is a prior record of prescribing by another licensed provider. Second, pharmacists may refill contraceptives only after recognizing that the patient had previously visited another clinician. The number of refills that pharmacists can prescribe is not limited by any protocol's restrictions. Third, before prescribing a contraceptive, pharmacists are required to have patients fill out the Minnesota Hormonal Contraceptive Self-Screening Questionnaire and evaluate their responses with them. This survey collects information about the patient's (1) personal data, (2) previous use, adverse reaction, and contraindication to contraceptives, and (3) medical history. ${ }^{3}$ Fourth, pharmacists are required to measure the patient's blood pressure and follow the Minnesota Board of Pharmacy Standard Procedures Algorithm for Prescribing of Contraceptives ${ }^{4}$ in order to confirm indication, contraindication, or referral for contraceptives. Fifth, pharmacists are required to train patients on contraceptive self-administration, provide them counseling on drug use and management, and release them a written prescription record. The prescription of contraceptives cannot be delegated to another person and needs to be recorded in a written or electronic form. ${ }^{2}$

The mandates related to pharmacist prescribing of contraceptives in Minnesota are similar to that enacted by other states (Table 1). At the time of this writing, thirteen states, including Minnesota and the District of Columbia have enacted policies that allow pharmacists to prescribe contraceptives outside of a collaborative practice agreement. ${ }^{5}$ Oregon was the first state to implement this regulation in January 2016, followed by California in April 2016. ${ }^{6-8}$ Unlike Minnesota, California does not require contraceptive prescribing training for pharmacists who graduate from a 
pharmacy school in California in or after 2014. All other states, except Idaho, have defined training requirements for contraceptive pharmacist-prescribing. Oregon is the only state that has the same restrictions on patient age as Minnesota; Colorado, Utah, and West Virginia allow pharmacists to prescribe contraceptives only to patients of 18 years of age or older, while the other states do not have restrictions on patient age. Many other states use protocols for contraceptive pharmacist-prescribing as Minnesota and require patients' selfscreening and blood pressure measurement. However, in addition to not requiring these assessments, some states do not mandate pharmacists to follow a standardized prescribing algorithm or provide patient. ${ }^{6}$ Similar to Minnesota, other states' protocols do not mention restrictions on the number of refills that pharmacists can prescribe.

Table 1: Overview of states with enacted regulations on contraceptive pharmacist prescribing

\begin{tabular}{|c|c|c|c|c|c|c|c|}
\hline State & $\begin{array}{l}\text { Start } \\
\text { date }^{a}\end{array}$ & $\begin{array}{c}18 \text { years or } \\
\text { older }\end{array}$ & $\begin{array}{l}\text { Formulation } \\
\text { restrictions }^{b}\end{array}$ & $\begin{array}{l}\text { Training/ } \\
\text { education }\end{array}$ & $\begin{array}{l}\text { Patient self- } \\
\text { screening }\end{array}$ & $\begin{array}{l}\text { Prescribing } \\
\text { algorithm }^{c}\end{array}$ & $\begin{array}{c}\mathrm{BP} \\
\text { measurement }\end{array}$ \\
\hline$C A^{6,9,10}$ & 2016 & & & $x^{d}$ & $x$ & & $x^{e}$ \\
\hline $\mathrm{CO}^{6}$ & 2017 & $x$ & $X^{f}$ & $x$ & $x$ & $x$ & $x$ \\
\hline$D C^{6,11}$ & 2018 & & $x^{g}$ & $x$ & $x$ & & \\
\hline$I D^{6, h}$ & 2019 & & & & & & \\
\hline $\mathrm{HI}^{6}$ & 2017 & & & $x$ & $x$ & & \\
\hline $\mathrm{MD}^{6,13}$ & 2019 & & & $x$ & $x$ & $x$ & $x$ \\
\hline $\mathrm{MN}^{1-4}$ & 2020 & $X^{i}$ & & $x$ & $x$ & $x$ & $x$ \\
\hline $\mathrm{NH}^{14,15}$ & 2018 & & $x^{g}$ & $x$ & $X^{j}$ & $x^{k}$ & \\
\hline $\mathrm{NM}^{6,13,16}$ & 2017 & & & $x$ & $x$ & & $x$ \\
\hline $\mathrm{OR}^{6,17,18}$ & 2016 & $x^{i}$ & & $x$ & $x$ & $x$ & $x$ \\
\hline $\mathrm{UT}^{6}$ & 2019 & $x$ & $x^{g}$ & $x$ & $x$ & $x$ & $x$ \\
\hline $\mathrm{VA}^{18,19}$ & 2021 & $x$ & & $x$ & $x$ & $x$ & $x$ \\
\hline$W^{21-23}$ & 2020 & $x$ & & $x$ & $x$ & & \\
\hline $\begin{array}{l}{ }^{\mathrm{a}} \text { Date th } \\
{ }^{\mathrm{b}} \text { No res } \\
\text { formula } \\
{ }^{\mathrm{c}} \text { Pharm } \\
{ }^{\mathrm{d}} \text { not rec } \\
\mathrm{e}^{\mathrm{e}} \text { if comb } \\
{ }^{\mathrm{f}} \text { Oral an } \\
{ }^{\mathrm{g}} \text { Oral, tr } \\
{ }^{\mathrm{h}} \text { Idaho } \\
\text { encoura } \\
{ }^{\mathrm{i}} \text { if a pati } \\
\text { provider } \\
\text { j Pharma } \\
\mathrm{k} \text { Protor }\end{array}$ & $\begin{array}{l}\text { indicat } \\
\text { ctions ir } \\
\text { ns } \\
\text { ists are } \\
\text { red for } \\
\text { ed horr } \\
\text { transde } \\
\text { isderma } \\
\text { licy crit } \\
\text { d }^{12} \\
\text { it is und } \\
\text { re requ } \\
\text { sts shou } \\
\text { develor }\end{array}$ & $\begin{array}{l}\text { en pharmacis } \\
\text { s that pharm } \\
\text { ed to follow a } \\
\text { acists who gr } \\
\text { contraceptive } \\
\text { rescribing on } \\
\text { nal prescribir } \\
\text { not represer } \\
\text { age of } 18, \text { co }\end{array}$ & $\begin{array}{l}\text { can start provi } \\
\text { ists can prescr } \\
\text { algorithm for } \\
\text { duate from a p } \\
\text { are requested } \\
\text { only } \\
\text { legal restrictio } \\
\text { irmed evidenc } \\
\text { mechanism }\end{array}$ & $\begin{array}{l}\text { g this servic } \\
\text { oral, transs } \\
\text { scribing con } \\
\text { macy schoo } \\
\text { recommenc }\end{array}$ & e prescribing, & ut practice gu & $\begin{array}{l}\text { lelines that are } \\
\text { lother licensed }\end{array}$ \\
\hline
\end{tabular}




\section{Benefits of the policy}

Allowing Minnesota's pharmacists to prescribe contraceptives has the potential to expand access to contraceptive services for patients. Studies have shown that many patients have a higher frequency of visits to community pharmacies than to primary care physicians which makes them a readily accessible source for health care. ${ }^{24}$ The accessibility of community pharmacists can benefit patients receiving contraceptive prescriptions and enhance contraceptive adherence. ${ }^{25}$ Pharmacists already reported their intention to prescribe contraceptive in their practice. ${ }^{26}$ The willingness of pharmacists to provide this service is demonstrated across states that have adopted these policies. A study conducted in one of the earliest states to adopt this service (California) showed that in 2017, 11.1\% of community pharmacies provided contraceptive prescribing services. ${ }^{7}$ Although an $11 \%$ participation rate may seem low, with more than 5,000 community pharmacies in California, ${ }^{27}$ this represents more than 500 additional contraceptive prescribing locations previously unable to provide this service. In San Francisco, 21 of 113 community pharmacies in the city provided pharmacist-prescribed contraceptives. ${ }^{25}$ In Oregon, the first state to adopt contraceptive prescribing by pharmacies, a survey delivered prior to the policy being enacted showed that $39 \%$ of pharmacists planned to provide contraceptive prescribing. ${ }^{28}$ Although only $39 \%$ of pharmacists planned to provide contraceptive prescribing, this represents a large increase in access for patients who were previously unable to use pharmacies to obtain this service. In addition, the availability of pharmacists to prescribe contraceptives as measured by the receipt of formal training required for the service increased from $19 \%$ to $63 \%$ of all state zip codes over a 6 month period since the law was established. ${ }^{18}$ The results from these states suggest that Minnesota also has the potential for pharmacists in the community to provide contraceptive prescribing, which would increase access to this service.
The willingness of pharmacists to provide contraceptive prescribing services has the potential to translate into increased access to contraceptive services for patients. A national survey showed that women facing issues obtaining contraceptive prescriptions have reported a willingness to use pharmacies to obtain contraceptives. ${ }^{29}$ Another study that interviewed women who received pharmacist-prescribed contraceptives reported that women were willing to continue to receive this service in pharmacies. ${ }^{30}$ This willingness has translated into greater access to this service in states that have expanded prescribing. In Oregon, between 2016 and 2017, pharmacists prescribed $10 \%$ of all the new prescriptions for contraceptives administered orally or transdermally to Medicaid enrollees. ${ }^{31}$ Contraceptive pharmacist-prescribing does not involve only new prescriptions. A study conducted in Oregon and California estimated that $91 \%$ of patients who visited a pharmacy to receive a contraceptive prescription already used contraceptives. ${ }^{32}$ In addition to increased access, the policy allowing for pharmacist-prescribed contraceptives can be effective in other aspects (Table 2 ).

Facilitating access to contraceptives can decrease unintended pregnancy, reduce costs, and improve outcomes. ${ }^{33}$ In Oregon, the expansion of prescribing to pharmacists has been suggested to translate into 51 avoidable unintended pregnancies and \$1.6 million in savings to the state Medicaid program over a two year period since the policy was implemented. ${ }^{34}$ It should also be noted that in comparison to a physician appointment, contraceptive prescribing by a pharmacist may be a more costeffective option for patients. ${ }^{25}$ In California, many pharmacies waive prescribing fees for patients to increase access to this service. $^{25}$

Table 2: Potential benefits and limitations of contraceptive pharmacist prescribing

\begin{tabular}{|c|c|}
\hline Benefits & Limitations \\
\hline Expand pharmacy services at community practice sites & Lack of staff, time, and space in pharmacy \\
\hline Improve access to contraceptives & $\begin{array}{l}\text { Lack of reimbursement for the service that the } \\
\text { pharmacist provides }\end{array}$ \\
\hline $\begin{array}{l}\text { Facilitate the process to obtain prescribed } \\
\text { contraceptives (for both new and refill prescriptions) }\end{array}$ & Lack of coverage for the patient \\
\hline Reduce unintended pregnancies and save health costs & Patients' safety concerns about pharmacists' training \\
\hline $\begin{array}{l}\text { Offer a more cost-effective service for patients in } \\
\text { comparison to a physician's appointment }\end{array}$ & Out-of-pocket fees for the patient \\
\hline $\begin{array}{l}\text { Engage pharmacists in a patient-centered care } \\
\text { practice }\end{array}$ & $\begin{array}{l}\text { Safety concerns due to patients' screening, obtaining } \\
\text { records, and contraceptives' adverse reactions and } \\
\text { self-administration procedures }\end{array}$ \\
\hline
\end{tabular}




\section{Limitations of the policy}

While allowing Minnesota pharmacists to prescribe contraceptives has the potential to expand this service to patients, significant challenges abound this policy which may prevent these benefits from being fully recognized. A key barrier to the adoption of contraceptive prescribing by pharmacists is adequate reimbursement for the time spent delivering this service. ${ }^{6,7,26,35}$ A study conducted in Oregon reported that pharmacists spend an average of 26 minutes providing contraceptive prescriptions for each patient seen. ${ }^{18}$ The time needed to comply with regulatory guidelines associated with prescribing contraceptives may not be commensurate with the reimbursement available to pharmacies for providing this service. In a study that conducted interviews in New Mexico, pharmacists reported uncertainty with the reimbursement associated with this service and believed that they do not receive payment for the majority of the work involved in the provision of contraceptive prescriptions. ${ }^{35}$ In some cases, this service may not be paid for at all. A study analyzing 676 separate visits of contraceptive pharmacist-prescribing in California and Oregon reported that $16 \%$ of the patients did not have insurance. ${ }^{32}$ In addition, pharmacists prescribing contraceptives may require patients to pay a fee. ${ }^{7,32}$ A study conducted in New Mexico reported that most of the pharmacists studied think that a fee between $\$ 30$ and $\$ 60$ may fairly compensate them for the service they provide. ${ }^{35}$ However, this fee may be charged out of pocket for the patients. ${ }^{25,32}$ California pharmacists reported that patients may not know that pharmacists charge a fee for contraceptive prescribing and that patients may not be able to pay for it. ${ }^{25} \mathrm{As}$ a result, the pharmacist's fee charged out of pocket for patients may decrease accessibility to this service. ${ }^{32}$ To overcome some of these challenges, pharmacists should align the provision of contraceptive prescribing with business models that provide reimbursement for this service. ${ }^{6}$ Pharmacies located in states that reimburse contraceptive prescribing should focus on identifying state-designated payers that pay for this service and building billing and reimbursement agreements with them. ${ }^{31}$ Other challenges to the prescribing of contraceptives that may limit the provision of this service include lack of staffing, $, 6,28$ time, ${ }^{6,25,26}$ space $^{25}$ liability, ${ }^{36}$ resistance from physicians, ${ }^{36}$ and implementation.

Another challenge may be represented by the policy's restriction on age. Minnesota requires patients under the age of 18 to show evidence of a previous contraceptive prescription obtained from another licensed provider. This presents barriers to accessing and scheduling appointments with providers, associated transportation, and other costs that may prevent these patients from obtaining contraceptive prescriptions. ${ }^{37,38}$ Perceptions of pharmacist-prescribed contraceptives have been explored in patients under the age of 18 . These patients reported that the accessibility of pharmacies and their lack of scheduling requirements makes obtaining contraceptive prescriptions more convenient. Patients also acknowledged that pharmacists are the medication experts and are trained to provide this service. ${ }^{38}$ Pharmacies' accessibility and pharmacists' medication expertise may encourage people under the age of 18 to receive pharmacist-prescribed contraceptives and address the high rate of unintended pregnancy reported in this population. ${ }^{37}$

Another challenge that prevents the achievement of the benefits of contraceptive pharmacists prescribing is placing limits on a pharmacist's ability to prescribe refills. A study conducted in 2019 reported that pharmacists are more likely to prescribe 6 months or more of contraceptives supply at a time. $^{39}$ This practice is supported by the protocol on contraceptive prescribing used in Minnesota, which does not indicate limitations on the number of refills that pharmacists can prescribe. As indicated in the protocol, pharmacists in Minnesota are required to provide the patient with a written record that includes the number of refills approved. ${ }^{3}$ Other states allow flexibility in the supply of pharmacist-prescribed contraceptives. Pharmacists practicing in the District of Columbia can prescribe a 12-month supply of contraceptives after proper patients' assessments and screenings are conducted. ${ }^{11}$ Allowing pharmacists to prescribe 6 months or more of contraceptive supply has the potential to improve contraceptive adherence and decrease unintended pregnancies and should be supported across states' policies. ${ }^{39}$

Expanding access through pharmacist-prescribing places Minnesota pharmacists in a position to ensure safety in the prescribing and use of contraceptives. Patients questioned the safety of contraceptives and reported concerns about their adverse events. ${ }^{29}$ Patient hesitancy may discourage them from accessing a pharmacy to obtain contraceptive services. Patients need to be assured that pharmacists are trained to be the experts in managing drugs' uses and that pharmacistprescribed contraceptives are safe. ${ }^{6}$ A study conducted in 21 states in the United States reported pharmacists' opinions about the safety of pharmacist-prescribed contraceptives. ${ }^{40}$ In this study, the majority of pharmacists rated safety concerns including patients' screening, obtaining records, and contraceptives' adverse reactions and self-administration procedures as very important aspects of the service. The study showed that there was not a significant difference in safety concerns between pharmacist respondents who were allowed or not allowed by their local state policies to prescribe contraceptives. ${ }^{40}$ In addition, patients may need to be educated on the training that pharmacists received to prescribe contraceptives. ${ }^{6}$ While 11 out of 13 states require specific training for pharmacists prescribing contraceptives, Idaho exempts training for all pharmacists and California exempts training for recent pharmacist graduates of a California school. ${ }^{6}$ Even though these two states waive training requirements, the competency of pharmacists in contraceptive use is apparent. In 2016, the American College of Clinical Pharmacy Educational Affairs Committee released guidance on pharmacotherapy content in pharmacy curriculum and assigned a Tier 1 status to contraceptive competency; ${ }^{41}$ Tier 1 indicates that "students 
receive education and training on this topic to prepare them to provide collaborative, patient-centered care on graduation and licensure." To offer this service, pharmacists should demonstrate their abilities to manage contraceptive use and identify potential adverse events and contraindications. A study conducted in Oregon measured the safety of contraceptives prescribed by pharmacists by assessing the percentage of patients receiving these medications when contraindicated; ${ }^{31}$ the study results showed that pharmacists abided to the prescribing algorithm and achieved a level of safety comparable to other providers when prescribing contraceptives. ${ }^{31}$

\section{Implications of the policy}

Although the legislation recently passed in Minnesota has the potential to significantly improve access to services for women needing contraceptive services in the state, the policy as enacted may be insufficient to ensure broad adoption by pharmacists. A significant limitation of this policy relates to insufficient reimbursement for the time spent providing this service. Without a specific reimbursement mandate, the time needed to fulfill the additional requirements of the service, the need for support staffing, and the willingness to work this service into existing workflow may be hampered. Adequate reimbursement is needed to support contraceptive access, ${ }^{18,31}$ improve outcomes, ${ }^{18}$ and promote the sustainability of this service. ${ }^{28}$ In Oregon, pharmacists can bill insurance for the service offered when they prescribe contraceptives, in addition to billing for the contraceptive drugs dispensed. ${ }^{17}$ Moreover, Oregon reimburses pharmacists for visits related to contraceptive prescribing under Medicaid. ${ }^{18}$ Contraceptive pharmacist-prescribing is also covered by Medicaid in California, which reimburses pharmacists approximately $\$ 45$ per service, ${ }^{42}$ and Maryland. ${ }^{6}$ Other states are making progresses toward the reimbursement of pharmacists' prescribing services. In 2020, New Mexico passed legislation that mandates insurance plans to provide reimbursement for pharmacists' prescribing services, including contraceptive prescribing, at rates similar to those received by physicians and nurse practitioners. ${ }^{16,43}$ In the same year, West Virginia passed legislation that requires insurance plans to provide payment for pharmacists' services including contraceptive prescribing if the service would have been paid if offered by another provider. ${ }^{22,23}$ States having policies that do not include payment for contraceptive pharmacist-prescribing reported limited implementation and discontinuation of this service. ${ }^{26}$

In addition to adequate reimbursement, giving pharmacist's provider status in federal programs like Medicaid and Medicare may yield additional benefits to contraceptive prescribing uptake by pharmacists. ${ }^{44}$ Although few Medicare patients are likely to need contraceptive services, federal policy enactment on issues like provider recognition often leads to broader policy uptake by other insurers. Minnesota should look to states like Oregon, who has been an early adopter with broad access to pharmacist prescribing of oral contraceptives, in developing policies to support reimbursement for this service. This may include exploring payers' reimbursement procedures for health care providers' services and aligning payments for contraceptive pharmacist-prescribing with current reimbursement models. ${ }^{31}$ As these services expand, the importance of studies directed at understanding the implementation of these services is important. ${ }^{31,32}$ So too are formal evaluations of patient involvement, ${ }^{18}$ and the health outcomes associated with this service. ${ }^{45}$ Future research comparing states' policies and reimbursement procedures for services that pharmacists offer and bill for as providers is needed to support contraceptive pharmacist-prescribing in Minnesota. Moreover, potential future research comparing outcomes of pharmacist-prescribed contraceptives between states that do and not reimburse pharmacists may provide data to support expanded reimbursement for this service.

Although the focus of this paper is on U.S. policies, it is worth mentioning that other countries, such as Canada, also allow pharmacist prescribing of oral contraceptives. ${ }^{46}$ The lessons of these other countries present a useful comparison to the Minnesota policy and its implications for patients and pharmacists.

\section{Conclusion}

With the recent passage of state legislation, Minnesota's pharmacists are now authorized to offer contraceptive prescribing. The authorization, similar to those granted by other states, may improve patients' access to contraceptives, decrease overall health costs, and improve outcomes. However, the effectiveness of this policy may be hindered by challenges including lack of staff, time, and payment. Minnesota policymakers should align this new authorization with current reimbursement models to support the sustainability of this service and explore opportunities to improve the patient care.

Conflicts of Interest: We declare no conflicts of interest or financial interests that the authors or members of their immediate families have in any product or service discussed in the manuscript, including grants (pending or received), employment, gifts, stock holdings or options, honoraria, consultancies, expert testimony, patents and royalties.

\section{References}

1. Anderson K. Pharmacists Authorized to Prescribe SelfAdministered Hormonal Contraceptives. University of Minnesota College of Pharmacy. https://www.pharmacy.umn.edu/sites/pharmacy.umn.edu /files/curbside q3 2020 final.pdf. Published 2020.

Accessed January 9, 2021.

2. Summary of 2020 Legislation. Minnesota Board of Pharmacy.

https://mn.gov/boards/assets/2020 Minnesota Legislation Affecting Pharmacy tcm21-440503.pdf. Published July 15, 2020. Accessed January 9, 2021. 
3. Pharmacist Prescribing Protocol Self-administered Hormonal Contraceptives. Minnesota Board of Pharmacy. https://mn.gov/boards/assets/Minnesota\%20Board\%20of \%20Pharmacy\%20Protocol\%20for\%20Pharmacist\%20Contr aceptive\%20Prescribing\%20Final\%20Draft tcm 21461180.pdf. Published October 19, 2020. Updated December 24, 2020. Accessed January 9, 2021.

4. Minnesota board of pharmacy standard procedures algorithm for prescribing of contraceptives. Minnesota Board of Pharmacy. https://mn.gov/boards/assets/Minnesota\%20Board\%20of \%20Pharmacy\%20Standard\%20Procedures\%20Algorithm\% 20for\%20RPh\%20Prescribing\%20of\%20Contraceptives tcm 21-461181.pdf. Published December 24, 2020. Accessed January 9, 2021.

5. Pharmacist Prescribing: Hormonal Contraceptives. National Alliance of State Pharmacy Associations.

https://naspa.us/resource/contraceptives/. Updated August 20, 2020. Accessed January 9, 2021.

6. Manmeet K, Honey J, Brooke G, Lynch S, Vest K, StewartLynch A, Weaver K. Hormonal contraception prescribing by pharmacists: 2019 update. J Am Pharm Assoc (2003). 2020;60(5):34-39. doi:10.1016/j.japh.2020.01.015

7. Gomez AM. Availability of Pharmacist-Prescribed Contraception in California, 2017. JAMA. 2017;318(22):2253-2254. doi:10.1001/jama.2017.15674

8. Ross, M. Pharmacists in California Can Prescribe Birth Control. Pharmacy Times. https://www.pharmacytimes.com/view/pharmacists-incalifornia-set-to-prescribe-birth-control Accessed on May 11, 2021.

9. Self-Administered Hormonal Contraception Protocol for Pharmacists. California State Board of Pharmacy. https://www.pharmacy.ca.gov/publications/hormonal con traception protocol rphs.pdf. Accessed January 12, 2021.

10. AB-1114 Medi-Cal: pharmacist services. California Legislative Information. https://leginfo.legislature.ca.gov/faces/billTextClient.xhtml ?bill id=201520160AB1114. Accessed January 12, 2021.

11. An Act D.C. ACT 22-246. Council of the District of Columbia. https://lims.dccouncil.us/downloads/LIMS/37359/Signed A ct/B22-0106-SignedAct.pdf. Accessed January 9, 2021.

12. Adams AJ. Pharmacist Prescriptive Authority: Lessons from Idaho. Pharmacy (Basel). 2020;8(3):112. Published $2020 \mathrm{Jul}$ 8. doi:10.3390/pharmacy8030112

13. Mitchell M, Stauffenberg C, Vernon V, Mospan CM, Shipman AJ, Rafie S. Opposition to Pharmacist Contraception Services: Evidence for Rebuttal. Pharmacy (Basel). 2020;8(4):176. doi:10.3390/pharmacy8040176

14. Title XXX Occupations and Professions. The General Court Of New Hampshire. http://www.gencourt.state.nh.us/rsa/html/XXX/318/31847-I.htm Accessed on April 3, 2021.

15. DeWitt E. House passes bill allowing pharmacists to prescribe contraceptives. Concord Monitor. https://www.concordmonitor.com/New-Hampshire-Housepasses-bill-to-allow-pharmacists-to-prescribecontraceptives-16371569 Published March 22, 2018. Accessed on April 3, 2021.
16. Nessel J. New Mexico Bill Requires Parity Reimbursement for Pharmacy Services. Pharmacy Times.

https://www.pharmacytimes.com/news/new-mexico-billrequires-parity-reimbursement-for-pharmacy-services. Published March 6, 2020. Accessed January 9, 2021.

17. Rodriguez MI, Anderson L, Edelman AB. Prescription of Hormonal Contraception by Pharmacists in Oregon: Implementation of House Bill 2879. Obstet Gynecol. 2016;128(1):168-170. doi:10.1097/AOG.0000000000001474

18. Rodriguez MI, Biel FM, Swartz JJ, Anderson L, Edelman AB. Pharmacists' experience with prescribing hormonal contraception in Oregon. J Am Pharm Assoc (2003). 2018;58(6):608-613. doi:10.1016/j.japh.2018.06.020

19. Virginia Board of Pharmacy Pharmacist Hormonal Contraceptive Statewide Protocol (Excluding Emergency Contraception). Virginia Department of Health Professions. https://www.dhp.virginia.gov/pharmacy/docs/protocols/Ph armacist\%20routine\%20contraceptive\%20statewide\%20pro tocol-9-9-2020.docx Accessed on April 3, 2021.

20. Virginia Algorithm for Pharmacists to Prescribe Routine Hormonal Contraceptives. Virginia Department of Health Professions.

https://www.dhp.virginia.gov/pharmacy/docs/protocols/Al gorithm\%20for\%20Pharmacists\%20to\%20Prescribe $\% 20$ Rou tine\%20Hormonal\%20Contraceptives-9-9-2020.docx Accessed on April 3, 2021.

21. Coyne C. Pharmacist-prescribed birth control not likely before 2020 in WV. Charleston Gazette-Mail. https://www.wvgazettemail.com/news/health/pharmacistprescribed-birth-control-not-likely-before-2020-inwv/article 23565b93-2e2e-599c-9fe3-c28a15a581fa.html. Published July 6, 2019. Accessed January 12, 2021.

22. Nessel J. West Virginia Bill to Expand Pharmacy Prescribing, Reimbursement. Pharmacy Times.

https://www.pharmacytimes.com/news/west-virginia-billto-expand-pharmacy-prescribing-reimbursement. Published March 17, 2020. Accessed January 9, 2021.

23. 2020 Regular Session Enrolled Committee Substitute for Senate Bill 787. West Virginia Legislature.

http://www.wvlegislature.gov/Bill Text HTML/2020 SESSI ONS/RS/signed bills/senate/SB787\%20SUB1\%20ENR signe d.pdf. Accessed January 9, 2021.

24. Berenbrok LA, Gabriel N, Coley KC, Hernandez I. Evaluation of Frequency of Encounters With Primary Care Physicians vs Visits to Community Pharmacies Among Medicare Beneficiaries. JAMA Netw Open. 2020;3(7):e209132. Published 2020 Jul 1. doi:10.1001/jamanetworkopen.2020.9132

25. Chen L, Lim J, Jeong A, Apollonio DE. Implementation of hormonal contraceptive furnishing in San Francisco community pharmacies. J Am Pharm Assoc (2003). 2020;60(6):963-968. doi:10.1016/j.japh.2020.07.019

26. Vu K, Rafie S, Grindlay K, Gutierrez H, Grossman D. Pharmacist Intentions to Prescribe Hormonal Contraception Following New Legislative Authority in California. J Pharm Pract. 2019;32(1):54-61. doi:10.1177/0897190017737897

27. NCPA 2016 Digest. National Alliance of State Pharmacy Associations. http://www.ncpa.co/pdf/digest/2016/2016ncpa-digest-spon-cardinal.pdf. Accessed on June 5, 2021. 
28. Rodriguez MI, McConnell KJ, Swartz J, Edelman AB. Pharmacist prescription of hormonal contraception in Oregon: Baseline knowledge and interest in provision. J Am Pharm Assoc (2003). 2016;56(5):521-526. doi:10.1016/j.japh.2016.05.003

29. Landau SC, Tapias MP, McGhee BT. Birth control within reach: a national survey on women's attitudes toward and interest in pharmacy access to hormonal contraception. Contraception. 2006;74(6):463-470. doi:10.1016/j.contraception.2006.07.006

30. Gardner JS, Downing DF, Blough D, Miller L, Le S, Shotorbani S. Pharmacist prescribing of hormonal contraceptives: results of the Direct Access study. J Am Pharm Assoc (2003). 2008;48(2):212-226. doi:10.1331/JAPhA.2008.07138

31. Anderson L, Hartung DM, Middleton L, Rodriguez MI. Pharmacist Provision of Hormonal Contraception in the Oregon Medicaid Population [published correction appears in Obstet Gynecol. 2020 Jan;135(1):222]. Obstet Gynecol. 2019;133(6):1231-1237. doi:10.1097/AOG.0000000000003286

32. Lu S, Rafie S, Hamper J, Strauss R, Kroon L. Characterizing pharmacist-prescribed hormonal contraception services and users in California and Oregon pharmacies. Contraception. 2019;99(4):239-243. doi:10.1016/j.contraception.2018.12.002

33. Access to Contraception. The American College of Obstetricians and Gynecologists. https://www.acog.org/clinical/clinicalguidance/committee-opinion/articles/2015/01/access-tocontraception. Accessed January 9, 2021.

34. Rodriguez MI, Hersh A, Anderson LB, Hartung DM, Edelman $A B$. Association of Pharmacist Prescription of Hormonal Contraception With Unintended Pregnancies and Medicaid Costs [published correction appears in Obstet Gynecol. 2019 Jul;134(1):182]. Obstet Gynecol. 2019;133(6):12381246. doi:10.1097/AOG.0000000000003265

35. Herman A, McCauley G, Thaxton L, Borrego M, Sussman AL, Espey E. Perspectives on prescribing hormonal contraception among rural New Mexican pharmacists. J Am Pharm Assoc (2003). 2020;60(5):57-63. doi:10.1016/j.japh.2020.02.026

36. Eckhaus LM, Ti AJ, Curtis KM, Stewart-Lynch AL, Whiteman MK. Patient and pharmacist perspectives on pharmacistprescribed contraception: A systematic review. Contraception. 2021;103(2):66-74. doi:10.1016/j.contraception.2020.10.012

37. Wilkinson TA, Miller C, Rafie S, Landau SC, Rafie S. Older teen attitudes toward birth control access in pharmacies: a qualitative study. Contraception. 2018;97(3):249-255. doi:10.1016/j.contraception.2017.11.008
38. Meredith AH, Vahary EB, Wilkinson TA, Meagher CG, Vielott T, Ott MA. Adolescents' Perceptions of Contraception Access through Pharmacies. Pharmacy (Basel). 2020;8(2):53. Published 2020 Mar 28. doi:10.3390/pharmacy8020053

39. Rodriguez MI, Edelman AB, Skye M, Anderson L, Darney BG. Association of Pharmacist Prescription With Dispensed Duration of Hormonal Contraception. JAMA Netw Open. 2020;3(5):e205252. Published 2020 May 1. doi:10.1001/jamanetworkopen.2020.5252

40. Rafie S, Cieri-Hutcherson NE, Frame TR, et al. Pharmacists' Perspectives on Prescribing and Expanding Access to Hormonal Contraception in Pharmacies in the United States [published online ahead of print, 2019 Aug 7]. J Pharm Pract. 2019;897190019867601. doi:10.1177/0897190019867601

41. Lynch SE, Griffin B, Stewart-Lynch A, Vest KM. Effect of a Simulated Activity on Student Ability, Preparedness, and Confidence in Applying the Pharmacists' Patient Care Process to Contraceptive Prescribing. Pharmacy (Basel). 2020;8(3):146. Published 2020 Aug 17. doi:10.3390/pharmacy8030146

42. Kritz, F. Pharmacists Can Now Prescribe Birth Control, But Few Do. California Health Report. https://www.calhealthreport.org/2019/02/15/pharmacistscan-now-prescribe-birth-control-but-few-do-\%EF\%BB\%BF/. Accessed on June 8, 2021.

43. Pharmaceutical Service Reimbursement Parity. New Mexico Legislature. https://www.nmlegis.gov/Sessions/20\%20Regular/final/HB 0042.pdf. Accessed on June 6, 2021.

44. Minnesota Legislative Preview: Hot Issues for the 2020 Session. Minnesota Pharmacists Association. https://cdn.ymaws.com/www.mpha.org/resource/resmgr/f iles/MPhA-Magazine-Winter-2020.pdf. Accessed January 9, 2021.

45. O'Connell MB, Samman L, Bailey T, King L, Wellman GS. Attitudes of Michigan Female College Students About Pharmacists Prescribing Birth Control in a Community Pharmacy. Pharmacy (Basel). 2020;8(2):99. doi:10.3390/pharmacy8020099

46. Contraceptive Prescribing. Canadian Pharmacists Association. https://www.pharmacists.ca/cphaca/assets/File/cpha-on-the-issues/ContraceptionInfographic.pdf Accessed on June 5, 2021. 